\title{
Philosophiques
}

\section{Note sur le puzzle de Kripke}

\section{Daniel Laurier}

Volume 15, numéro 1, printemps 1988

URI : https://id.erudit.org/iderudit/027032ar

DOI : https://doi.org/10.7202/027032ar

Aller au sommaire du numéro

Éditeur(s)

Société de philosophie du Québec

ISSN

0316-2923 (imprimé)

1492-1391 (numérique)

Découvrir la revue

Citer cet article

Laurier, D. (1988). Note sur le puzzle de Kripke. Philosophiques, 15(1), 31-39.

https://doi.org/10.7202/027032ar

\section{Résumé de l'article}

Je soutiens que Kripke n'a pas réussi à montrer que certains principes plausibles gouvernant l'attribution de croyances, tels que les principes de décitation et de traduction, pouvaient nous conduire à attribuer des croyances de dicto contradictoires à un sujet réfléchi et linguistiquement compétent sans présupposer une théorie descriptive des noms propres ou des termes désignant des espèces naturelles. Les cas décrits par Kripke se réduisent à des variantes du problème de Quine concernant les croyances de re ou à des variantes du puzzle de Mates concernant l'intersubstiruabilité des synonymes dans les contextes de croyance. d'utilisation que vous pouvez consulter en ligne.

https://apropos.erudit.org/fr/usagers/politique-dutilisation/ 


\title{
NOTE SUR LE PUZZLE DE KRIPKE
}

\author{
par Daniel Laurier
}

\begin{abstract}
RÉSUMÉ. Je soutiens que Kripke n'a pas réussi à montrer que certains principes plausibles gouvernant l'attribution de croyances, tels que les principes de décitation et de traduction, pouvaient nous conduire à attribuer des croyances de dicto contradictoires à un sujet réfléchi et linguistiquement compétent sans présupposer une théorie descriptive des noms propres ou des termes désignant des espèces naturelles. Les cas décrits par Kripke se réduisent à des variantes du problème de Quine concernant les croyances de re ou à des variantes du puzzle de Mates concernant l'intersubstituabilité des synonymes dans les contextes de croyance.
\end{abstract}

ABSTRACT. I claim that Kripke failed to show that certain plausible principles governing belief ascription, such as the disquotation and translation principles, could lead one to ascribe contradictory de dicto beliefs to a reflexive and linguistically competent agent without presupposing a descriptive theory of names or natural kind terms. Kripke's examples are nothing but variants either of Quine's problem about de re beliefs, or of Mates' puzzle concerning the interchangeability of synonyms in belief contexts.

J'ai soutenu dans Laurier (1986) ${ }^{1}$ que contrairement à ce que prétend Kripke le genre de situations qu'il décrit comme donnant lieu à un puzzle ne concerne pas des attributions de croyance de dicto mais des attributions de croyance de re, et qu'en ce sens son

1. Ceci est le texte à peine remanié de ma contribution à une table ronde sur la notion de croyance qui s'est tenue en mai 1986 à l'université de Montréal, dans le cadre du congrès annuel de la Société de philosophie du Québec. Les autres participants à cette table ronde étaient François Lepage, Claude Panaccio et Michel Seymour. Je renvoie les lecteurs qui n'auraient aucune connaissance du puzzle de Kripke au texte de M. Seymour, ou encore à Kripke (1979) ou Laurier (1985, 1986). Je remercie tout particulièrement Phil Hanson et Pascal Engel de leurs réactions critiques à Laurier (1986), qui m’ont conduit à la présente mise au point. 
soi-disant puzzle n'est pas différent, en principe, du cas classique discuté notamment par Quine concernant les attributions de croyance de re.

Quine souligne, en deux mots, qu'il est parfaitement possible que Ralph croie (de re) de Ortcutt qu'il est un espion et qu'il n'est pas un espion, sans pour autant se contredire, parce qu'il pense au même individu (à savoir Ortcutt) sous deux descriptions distinctes dont il ignore qu'elles sont codésignatives. Comme Quine et Kripke l'admettent tous les deux, attribuer de telles croyances de re à Ralph ce n'est pas lui attribuer des croyances contradictoires; et cela ne soulève donc pas de problème sérieux.

Mais selon Kripke, si on admet certains principes généraux à première vue assez plausibles on peut être amené à attribuer à un sujet réfléchi et linguistiquement compétent des croyances de dicto contradictoires, et c'est en cela que consiste (en principe) l'originalité du puzzle puisque alors on ne peut apparemment plus expliquer la situation en disant que le sujet en question a des croyances de re incompatibles mais des croyances de dicto consistantes.

Un autre aspect de la position de Kripke qui est pertinent pour la discussion est que selon lui le puzzle ne dépend d'aucune théorie particulière des noms propres; et en particulier, il ne présuppose ni une conception descriptiviste, selon laquelle le sens d'un nom propre serait celui d'une description définie associée à ce nom, ni une conception millienne, selon laquelle la valeur sémantique d'un nom ne serait rien d'autre que sa dénotation (i.e., le porteur du nom).

J'ai proposé dans Laurier (1986) un argument (que je ne répéterai pas mais que j'estime toujours valide) destiné à montrer que si le cas «Paderewski ${ }^{2}$ est bien un exemple du type de puzzle que Kripke a en tête, alors ce puzzle présuppose vraisemblablement la conception millienne. De là je concluais que puisqu'un énoncé

2. Ce cas est le suivant. Pierre entend parler du célèbre pianiste Paderewski, et plus tard, d'un homme politique du même nom. Convaincu quaucun homme politique ne peut avoir de talent musical, il en vient à accepter simultanément : «Paderewski est un grand pianiste» et «Paderewski n'est pas un grand pianiste». Mais il ignore qu'il n'y a qu'un Paderewski. 
comme «Pierre croit que Paderewski est un grand pianiste» n'a pas d'interprétation de dicto (distincte de l'interprétation de re) sous la conception millienne, le puzzle ne pouvait pas concerner les attributions de dicto.

Bien que cela ait été contesté notamment par P. Hanson, je persiste à croire que la conception millienne n'autorise pas vraiment une lecture de dicto des attributions de croyance. Mais dans ce qui suit je vais examiner une façon de donner du sens à cette idée, qui conduira néanmoins à la conclusion que les sujets dont parle Kripke n'ont pas de croyances de dicto contradictoires. Je crois utile de revenir sur cette question pour deux raisons. Premièrement, l'argument auquel je fais allusion aurait pu aussi bien (je le réalise maintenant) me conduire à la conclusion que le cas «Paderewski » n'est pas vraiment un exemple du puzzle en question, bien que Kripke le présente explicitement comme tel. Et deuxièmement, le puzzle concerne aussi bien, selon Kripke, les attributions de croyance qui utilisent des termes de sortes naturelles que celles qui utilisent des noms propres ; cela veut dire que s'il fallait s'en tenir à mon ancien raisonnement il faudrait aussi conclure que Kripke se trompe encore quand il prétend que son puzzle concerne aussi les termes de sortes naturelles. On devrait être conduit à cette conclusion parce qu'il n'y a pas de conception purement millienne (viable) des termes de sortes naturelles et qu'il y a certainement une interprétation de dicto d'un énoncé comme «Pierre croit que les ormes sont des arbres tropicaux $»$.

Dans ces conditions, il paraît plus charitable de supposer que c'est le cas «Paderewski » qui ne donne pas lieu à un puzzle (ou du moins un puzzle du même type) et que les autres exemples discutés par Kripke concernent donc bel et bien une lecture de dicto des attributions de croyance. C'est sous cette présupposition que je me propose de réexaminer les cas décrits par Kripke et de montrer que dans ce cas ou bien le puzzle n'existe pas, ou bien il concerne la lecture de re, ou bien il se réduit au puzzle de Mates ${ }^{3}$.

3. Ce puzzle consiste dans le fait que deux expressions syntaxiquement primitives et parfaitement synonymes ne sont apparemment pas intersubstituables salva veritate dans les contextes d'attitudes propositionnelles. Ainsi, à supposer que « $\mathrm{Grec}$ » et « Hellène » soient des synonymes parfaits, Pierre pourrait croire à la fois que les Grecs sont des menteurs et que les Hellènes ne sont pas des menteurs, sans se rendre compte de l'inconsistance. 
Dans ce dernier cas, le puzzle présupposerait une conception descriptive des noms.

Avant d'aborder cette question, je voudrais souligner que le cas «Paderewski » est introduit par Kripke dans le contexte d'un argument destiné à montrer qu'on ne pourrait pas dissoudre le puzzle en stipulant que deux noms différents ne sont jamais intertraduisibles (ou synonymes), ou en invoquant une théorie descriptive des noms propres qui impliquerait que deux noms ne sont jamais intertraduisibles. Même si cet argument n'est pas parfaitement convaincant (s'il est vrai que le puzzle doit concerner les attributions de croyance de dicto), il reste que Kripke a un autre argument en faveur de la même conclusion, à savoir que si on soutient que deux noms propres ne sont jamais intertraduisibles il faudra aussi soutenir que deux termes de sortes naturelles ne le sont jamais non plus (puisque le puzzle les concerne aussi). Or ce serait tout à fait inacceptable parce que c'est un «fait brut» qu'on accepte de traduire, par exemple, le mot «elm» par le mot «orme ».

Cela veut dire que si le puzzle de Kripke ne présuppose aucune conception particulière des noms propres, il exclut en tout cas certaines formes de théories descriptives, qui impliquent que deux noms différents ne sont jamais intertraduisibles (c'est le cas notamment de celle que Engel appelle la théorie «descriptivenominale », selon laquelle «Londres » signifierait « l'objet nommé "Londres"»).

En d'autres termes les seules théories descriptives sous lesquelles le puzzle de Kripke peut être formulé sont celles qui rendent justice au fait brut que «Londres » et «London » sont synonymes, i.e., intertraduisibles. La même chose vaut évidemment, a fortiori, pour les termes de sortes naturelles.

Le problème qui se pose maintenant est celui de donner du sens à l'idée que les exemples de Kripke concernent des attributions de croyance de dicto sans présupposer aucune théorie particulière de la signification des noms propres ou des termes de sortes naturelles. La difficulté vient de ce que la notion d'interprétation de dicto est extrêmement vague quand on la considère en dehors de tout contexte théorique particulier. La seule caractéristique d'une interprétation de dicto à laquelle je puisse penser et qui ne 
préjuge pas d'une solution particulière au puzzle de Kripke est qu'une telle interprétation doit relier le sujet de la croyance à une entité abstraite aủ moins en principe saisissable par l'intuition, i.e. à une entité dont il soit possible d'avoir une "connaissance directe ». Je ne pense pas que cette formulation soit très heureuse, mais elle devrait suffire pour mon propos et j'espère que les remarques suivantes aideront à en faire saisir l'esprit.

Cette caractérisation est d'abord motivée par le désir d'en éviter une autre, qui dirait qu'une interprétation de dicto d'un énoncé comme «Pierre croit que Londres est jolie» est une interprétation selon laquelle Pierre serait relié d'une certaine manière à la signification de la phrase «Londres est jolie ». Je veux éviter cette formulation parce qu'un millien pourrait prétendre que la signification de cette phrase n'est rien d'autre que l'état de choses que Londres est jolie (ou dans une autre terminologie, la proposition singulière composée de la ville de Londres et de la propriété d'être jolie). Mais dans ce cas l'énoncé «Pierre croit que Londres est jolie » n'aurait pas encore, selon moi, d'interprétation de dicto; ou plus exactement il ne pourrait plus y avoir de contraste entre une interprétation de re et une interprétation de dicto de cet énoncé, puisqu'il serait alors logiquement équivalent à «Pierre croit de Londres qu'elle est jolie».

Cela ne signifie pas que cet énoncé ne peut pas recevoir d'interprétation de dicto sous la conception millienne. Mais pour pouvoir discerner une interprétation de dicto il faut distinguer deux aspects de la signification, i.e., il faut admettre en plus des états de choses ou des conditions de vérité quelque chose comme des sens frégéens ou des contenus conceptuels. Dans le cas limite d'un énoncé comme «Pierre croit que Londres est jolie», où le nom «Londres » est considéré comme purement millien, l'interprétation de dicto mettrait Pierre en relation avec le contenu conceptuel de la phrase «Londres est jolie », c'est-à-dire (dans une autre terminologie) avec la fonction propositionnelle «... est jolie », puisque le nom «Londres » n'a pas dans cette hypothèse de contenu conceptuel ${ }^{4}$.

4. Ou en d'autres termes, la signification littérale du nom «Londres » n'est pas dans cette hypothèse un contenu conceptuel. 
Je pense maintenant avoir introduit tous les éléments nécessaires pour établir ma conclusion. Supposons que Pierre, un locuteur réfléchi qui a la compétence de l'anglais et du français, donne son assentiment aux six phrases suivantes :

(1) Londres est jolie.

(2) London is not pretty.

(3) Les ormes sont des arbres tropicaux.

(4) Elms are not tropical trees.

(5) Les philosophes sont des menteurs.

(6) Philosophers are not liars.

On peut alors inférer, à l'aide des principes de «décitation » («disquotation principle») et de traduction et du manuel de traduction de l'anglais au français, que :

(7) Pierre croit que Londres est jolie et que Londres n'est pas jolie.

(8) Pierre croit que les ormes sont des arbres tropicaux et que les ormes ne sont pas des arbres tropicaux.

(9) Pierre croit que les philosophes sont des menteurs et que les philosophes ne sont pas des menteurs.

La question est de savoir s'il y a un sens dans lequel l'un ou l'autre de ces trois énoncés attribue des croyances de dicto contradictoires à Pierre, et qui ne nous oblige pas à abandonner l'hypothèse que Pierre est un locuteur compétent et réfléchi de l'anglais et du français. Je pense qu'il y a effectivement un tel sens, mais qui serait inacceptable pour Kripke, parce qu'il réduirait son puzzle à une variante de celui de Mates et qu'il nie explicitement que les deux types de cas soient parallèles.

Considérons un terme quelconque $\mathrm{N}$, qui peut être aussi bien un nom propre, qu'un terme de sorte naturelle ou un terme purement général. Il me semble qu'il y a seulement trois possibilités à considérer concernant son contenu conceptuel, à savoir : i) ou bien il est nul (théorie millienne); ii) ou bien sans être nul il est néanmoins insuffisant pour déterminer sa dénotation, de sorte que deux termes avec le même contenu conceptuel pourraient alors avoir des dénotations distinctes (théorie putnamienne); iii) ou bien il n'est pas nul et il détermine sa dénotation (théorie frégéenne). 
Il importe ici de se rappeler que dans le cas où le contenu conceptuel détermine la dénotation, il faut néanmoins s'interdire de supposer que ce contenu est de nature à exclure qu'un terme distinct puisse avoir le même contenu conceptuel. Car autrement on serait amené à soutenir que les paires «Londres »/ «London», «orme»/«elm» et «philosophe»/ «philosopher» ne sont pas des paires de termes intertraduisibles.

Il ne reste plus qu'à examiner ce qui se passe dans chaque cas. Il est clair que si le contenu conceptuel est nul ou simplement insuffisant pour déterminer la dénotation alors aucun des trois énoncés (7)-(9) n'attribue de croyances de dicto contradictoires à Pierre. Car en effet, dans ces deux hypothèses, les contenus conceptuels respectifs de (1)-(2), (3)-(4) et (5)-(6) ne sont pas incompatibles puisqu'ils n'expriment alors pas une pensée complète. On n'a donc pas affaire, alors, à un puzzle concernant des attributions de croyance de dicto. S'il y a un problème, il vient simplement du fait que «Londres » et «London », « orme » et « elm », « philosophe » et « philosopher » sont respectivement codésignatifs; i.e., il concerne des attributions de croyance de re. Il semble donc que si on assume une théorie millienne des noms propres ou une théorie putnamienne des termes de sortes naturelles alors les exemples de Kripke ne conduisent pas à attribuer des croyances de dicto contradictoires à Pierre.

On pourrait maintenant avoir l'impression que je force un peu la note en admettant qu'une interprétation de dicto puisse relier un sujet à un contenu conceptuel «incomplet». Mais il semble bien que ce soit le prix à payer si on veut admettre que les attributions de croyance ont une interprétation de dicto (littérale) sous la théorie millienne et/ou sous la théorie putnamienne. D'autre part, ce prix n'est peut-être pas excessif, si on prend note de ce que l'expression «contenu conceptuel» est ici utilisée de façon plutôt métaphorique et pourrait éventuellement être remplacée par une notion purement syntaxique ou structurale (ainsi, au lieu de parler du contenu conceptuel d'une phrase, on pourrait éventuellement parler de sa place dans la structure logique de la langue). Il ne faudrait pas croire, enfin, que cette position implique que les contenus de pensée de Pierre sont eux-mêmes incomplets ; elle implique seulement que ceux-ci sont incomplètement spécifiés par l'interprétation de dicto (littérale) d'énoncés comme «Pierre 
croit que Londres est jolie » ou «Pierre croit que les ormes sont des arbres tropicaux $»$. Rien n'interdit de supposer, par exemple, que quand Pierre utilise le nom «Londres » il lui associe une description définie (possiblement une description métalinguistique du type «l'objet nommé "Londres"»). On pourrait alors parler de sa croyance de dicto privée, par opposition à sa croyance de dicto publique, qui elle serait incomplète.

Quoi qu'il en soit, il reste encore un cas à examiner pour compléter mon argumentation. C'est celui qui correspond à la théorie sémantique classique, d'inspiration frégéenne, où on suppose que chaque expression a un sens qui en détermine la référence. Je pense que les critiques de Kripke à l'endroit de la conception frégéenne des noms propres sont justifiées et qu'il est par conséquent douteux qu'on puisse trouver une description définie "purement qualitative » qui exprime le sens littéral d'un nom propre dans une langue publique. Il n'en reste pas moins que c'est seulement à cette condition qu'on pourrait dire que des énoncés comme «Londres est jolie » et «London is not pretty » ont des contenus conceptuels (i.e., des sens) incompatibles. En d'autres termes c'est seulement dans le cas où les phrases utilisées pour spécifier les contenus de croyance de Pierre ont un sens frégéen qu'on peut supposer que les phrases (7)-(9) lui attribuent des croyances de dicto contradictoires.

Mais dans ce cas, si Pierre est vraiment réfléchi et parfaitement compétent (linguistiquement) il semblerait qu'il ne puisse pas manquer de déceler, par simple réflexion, qu'il a des croyances de dicto contradictoires ; i.e., qu'il ne puisse pas donner son assentiment à la fois à (1) et (2) (ou (3) et (4), ou (5) et (6)). On n'arrive pas en d'autres termes à produire le puzzle désiré, c'est-à-dire à conclure que Pierre a des croyances de dicto contradictoires sans être en mesure de le reconnaître. La seule façon d'arriver à cette conclusion serait apparemment d'admettre que l'hypothèse que Pierre est un locuteur compétent de l'anglais et du français n'implique pas qu'il maîtrise effectivement le contenu conceptuel des expressions anglaises ou françaises. Il faudrait ainsi admettre que sa compétence linguistique peut être moins que parfaite et qu'il peut comprendre certaines expressions d'une façon qui s'écarte de leur sens littéral sans pour autant cesser d'être un locuteur compétent. 
Dans ces conditions, on pourrait admettre que Pierre a des croyances de dicto incompatibles, mais seulement dans le sens où on peut admettre qu'un locuteur du français réfléchi et compétent peut croire de dicto que les Grecs ne sont pas des Hellènes, bien que «Grecs » et « Hellènes » soient synonymes. Ainsi, le soi-disant puzzle de Kripke se réduirait alors non pas à une variante du problème de Quine, mais à une variante du puzzle de Mates. Et comme dans le cas du puzzle de Mates, il semble qu'on puisse résoudre la difficulté en distinguant entre les croyances de dicto publiques de Pierre (qui sont contradictoires) et ses croyances de dicto privées (qui doivent être consistantes).

Département de philosophie

Université du Québec à Trois-Rivières

\section{RÉFÉRENCES}

ALMOG, Joseph (1984) : «Would you Believe that?", Syntbese 58, 1-37.

ENGEL, Pascal (1986) : “Croyance et référence », à paraître.

KRIPKE, SAUL (1979): “A Puzzle about Belief», Margalit A. ed. (1979), 239-283.

LAURIER, Daniel (1985): “Noms propres et attributions de croyances", Cahiers de grammaire 10, 75-92.

(1986): «Names and Beliefs: A Puzzle Lost», The Philosophical Quarterly 36, 37-49.

LINSKY, Leonard ed. (1952): Semantics and the Philosophy of Language,

Urbana, University of Illinois Press.

MARGALIT, Avishai ed. (1979) : Meaning and Use, Dordrecht, Reidel.

MATES, Benson (1950) : «Synonymity », Linsky L. ed. (1952), 111-136. 